\title{
TÉCNICA DE CONTROLE PREDITIVO BASEADO EM MODELO APLICADA AO CONTROLE DE TENSÃO DE UM GERADOR SÍNCRONO - RESULTADOS EXPERIMENTAIS
}

\author{
Marcelo Nascimento Moutinho* \\ marcelo.moutinho@eletronorte.gov.br \\ Walter Barra Jr. ${ }^{\dagger}$ \\ walbarra@ufpa.br
}

\author{
Carlos Tavares da Costa Jr. ${ }^{\dagger}$ \\ cartav@ufpa.br \\ José Augusto Lima Barreiros ${ }^{\dagger}$ \\ barreiro@ufpa.br
}

${ }^{*}$ Centro de Tecnologia da Eletronorte - LACEN, Rod. Artur Bernardes, no 2172 - Miramar, CEP: 66115-000 Belém, Pará, Brasil

†Universidade Federal do Pará-UFPA, Rua Augusto Corrêa 01, Belém, PA, Brasil

\begin{abstract}
Model Based Predictive Control Technique Applied to Voltage Control of a Synchronous Generator - Experimental Results

This paper presents the results of the experimental evaluation of a digital self-tuning predictive control methodology applied to the voltage control of a small-scale energy generation system. A recursive estimator based on the well known least-squares method is used in the identification stage of the proposed controller. The stage for calculation of the signal control method is performed with the Generalized Predictive Controller (GPC) algorithm. The experimental evaluation was performed using step perturbations applied in different operating conditions of the studied power system. For comparison purposes, the results of the evaluation of a self-tuning controller using the pole-placement method for the control signal formulation and three digital controllers with fixed parameters also will be presented.
\end{abstract}

KEYWORDS: Adaptive Control, Recursive Estimation, Predictive Control.

Artigo submetido em 17/08/2011 (Id.: 1369)

Revisado em 08/11/2011, 26/01/2012

Aceito sob recomendação do Editor Associado Prof. Luis Fernando Alves Pereira

\section{RESUMO}

Neste trabalho serão apresentados os resultados da avaliação experimental de uma metodologia de controle digital preditivo auto-ajustável aplicada ao controle de tensão de um sistema de geração de energia de escala reduzida. Um estimador recursivo baseado no conhecido método de mínimos quadrados é utilizado na etapa de identificação do controlador preditivo proposto. A etapa de cálculo da lei de controle é realizada com o algoritmo Generalized Predictive Controller (GPC). A avaliação experimental foi realizada com testes de resposta ao degrau e rastreamento aplicados em diferentes condições operacionais do sistema de potência estudado. Para fins de comparação, também serão apresentados os resultados da avaliação de um controlador auto-ajustável que utiliza o método de alocação de pólos para a síntese do sinal de controle e três controladores digitais com parâmetros fixos.

PALAVRAS-CHAVE: Controle Adaptativo, Estimação Recursiva, Controle Preditivo.

\section{INTRODUÇÃO}

Sob o ponto de vista da engenharia de controle, um Sistema Elétrico de Potência (SEP) pode ser consi- 
derado um sistema dinâmico de grande escala com múltiplas-entradas e múltiplas-saídas (MIMO, acrônimo do termo originalmente cunhado em língua inglesa, Multiple-Inputs Multiple-Outputs) que apresenta relações fortemente não-lineares entre suas variáveis. Em fato esse tipo de sistema é formado por numerosos dispositivos de proteção e de controle com diversas características e velocidades de resposta (Sauer and Pai, 1998).

Sob o ponto de vista da teoria de sistemas dinâmicos, essa elevada complexidade também é uma característica inerente à concepção estrutural dos sistemas elétricos, já que eles podem exibir mudanças em seus parâmetros naturalmente ao longo do tempo de utilização e também conforme a sua condição operacional é alterada (Robak, 2009):

Para lidar com as peculiaridades dos SEP é necessario o desenvolvimento de sistemas de controle robustos e seguros. No caso dos Reguladores de Velocidade (RVs) e Reguladores Automáticos de Tensão (RATs), normalmente são utilizados controladores contínuos com parâmetros fixos projetados com técnicas lineares convencionais. Nesse tipo de projeto são utilizados modelos matemáticos lineares obtidos por meio de técnicas de linearização aplicadas a modelos não-lineares representativos do SEP (Larsen and Swann., 1981). Devido à natureza não-linear dos SEPs, esses controladores têm dificuldade em apresentar um bom desempenho uniforme em todas as condições operacionais já que seus parâmetros são sintonizados durante a fase de projetado para uma condição operacional conhecida satisfazendo muito bem os critérios de desempenho naquela condição onde o modelo linear do sistema de potência foi obtido.

O controle de um SEP de forma estável e confiável em várias condições operacionais também pode ser realizado por meio da utilização de estruturas de controle projetadas com base em técnicas avançadas de controle digital. Essa é a principal motivação para o desenvolvimento deste artigo que versará sobre o projeto e a avaliação experimental de estratégias de controle preditivo e adaptativo aplicadas à SEPs. Neste artigo essas estratégias serão referenciadas como Model Predictive Controllers (MPC) (Camacho and Bordons, 2004).

A principal contribuição desse trabalho é de natureza prática já que serão apresentados os resultados de estudos experimentais relacionados à implementação de um RAT digital adaptativo projetado com técnica MPC. Para fins de comparação, dois projetos de controladores adaptativos serão discutidos. O esquema de controle adaptativo a ser utilizado em ambos é o autoajustável indireto (Aström and Wittenmark, 1998). A versão recursiva do método de estimação de mínimos quadrados (Aguirre, 2004) será utilizada em ambos os projetos. A diferença principal entre os dois projetos propostos reside na metodologia de síntese do sinal de controle. O primeiro projeto utiliza o método de alocação de pólos (Landau, 1993) (Aström and Wittenmark, 1995), o mesmo que foi utilizado no projeto do controlador digital de tensão a parâmetros fixos descrito em (Moutinho, da Costa Jr. and Barra, 2006). O segundo projeto utiliza o algoritmo Generalized Predictive Controller (GPC) (Clarke et al., 1987a). Os controladores tiveram seus desempenhos avaliados por meio de testes comparativos realizados em um Sistema de Geração de Energia de Escala Reduzida (SGER). Está fora do escopo deste trabalho realizar uma análise formal da estabilidade dos controladores adaptativos projetados. Apesar de esse estudo de estabilidade não ter sido realizado, em nenhum dos experimentos realizados pelos autores foi verificado perda de estabilidade em malha fechada com os controladores adaptativos. Também não foram incluídos no escopo do trabalho os estudos detalhados sobre a robustez, rejeição de perturbações e/ou atenuação de ruídos do controlador preditivo proposto.

Este artigo está organizado da seguinte forma: a seção 2 trata de uma breve descrição dos algoritmos MPC; na seção 3 será apresentada uma possível formulação do algoritmo de identificação recursivo utilizado; na seção 4 serão descritas as duas técnicas de formulação de sinal de controle utilizadas: o GPC e a técnica de alocação de pólos; a seção 5 descreve os resultados experimentais. O desempenho dos controladores foi avaliado por meio de testes comparativos de resposta ao degrau e rastreamento realizados em diferentes condições operacionais do SGER; na seção 6 são apresentadas as conclusões do trabalho e as perspectivas de trabalhos futuros.

\section{MODEL PREDICTIVE CONTROLLERS - MPC}

MPC é a sigla utilizada para referenciar um grupo especial de algoritmos de controle que utiliza explicitamente um modelo dinâmico para predizer o comportamento do processo controlado em um horizonte de tempo futuro finito. O sinal de controle otimizado é sintetizado com base na minimização de um critério de desempenho e em um conjunto de restrições, adequadamente definidos para cada aplicação. O MPC está amplamente difundidos no meio acadêmico (Bravo and Normey-Rico, 2009) e em diversas aplicações na indústria de processos (Fontes and Mendes, 2009) e de geração de energia (Barra et al., 2005). Um importante membro da família de algoritmos MPC, o Generalized 
Predictive Controlller (GPC), foi proposto em 1987 por Clarke (Clarke et al., 1987a; Clarke et al., 1987b; Clarke and Zhang, 1987). Atualmente o GPC é considerado um dos algoritmos MPC mais popularmente utilizados em aplicações industriais.

Na próxima seção será descrito o modelo utilizado no procedimento de identificação recursiva utilizado nos dois controladores adaptativos propostos neste trabalho.

\section{TÉCNICAS DE IDENTIFICAÇÃO RE- CURSIVA.}

Os controladores adaptativos propostos neste trabalho utilizam um esquema do tipo auto-ajustável indireto, compostos de duas partes: identificação e cálculo da lei de controle. A primeira parte é responsável pela estimação recursiva dos coeficientes de um modelo paramétrico. Devido a sua robustez e simplicidade computacional, o Estimador Recursivo de Mínimos Quadrados (ERMQ) (Aström and Wittenmark, 1995), será utilizado neste trabalho. A seguinte estrutura, conhecida como modelo paramétrico CARIMA, será utilizada para representar o modelo do processo a ser controlado:

$$
A\left(q^{-1}\right) y(t)=q^{-d} B\left(q^{-1}\right) u(t)+C\left(q^{-1}\right) \frac{e(t)}{\Delta}
$$

onde $u(t)$ e $y(t)$ são, respectivamente, os valores dos sinais de entrada e saída da planta no instante discreto $t$, um múltiplo inteiro do intervalo de amostragem $T_{s} ; e(t)$ é um ruído descorrelacionado que corrompe o sinal de saída do modelo; $d$ é o atraso de transporte da entrada para a saída representado como um múltiplo inteiro de $T_{s} ; q^{-1}$ é o operador atraso discreto ${ }^{1}$; e $\Delta=1-q^{-1}$. Os polinômios $A\left(q^{-1}\right), B\left(q^{-1}\right)$ e $C\left(q^{-1}\right)$ são da seguinte forma:

$$
\begin{gathered}
A\left(q^{-1}\right)=1+a_{1} q^{-1}+\ldots+a_{n_{a}} q^{-n_{a}} \\
B\left(q^{-1}\right)=b_{0}+b_{1} q^{-1}+\ldots+b_{n_{b}} q^{-n_{b}} \\
C\left(q^{-1}\right)=1+c_{1} q^{-1}+\ldots+c_{n_{c}} q^{-n_{c}}
\end{gathered}
$$

sendo $n_{a}, n_{b}$ e $n_{c}$ são o número de regressores da saída, da entrada e do ruído, respectivamente. O polinômio $C\left(q^{-1}\right)$ pode ser considerado o componente de uma perturbação externa que afeta a saída $y(t)$ (neste caso precisa ser estimado), ou pode ser interpretado como um observador polinomial para as predições das saídas (neste outro caso, faz parte dos parâmetros do projeto).

A próxima seção é devotada ao estudo das duas técnicas de síntese de sinal de controle utilizadas neste trabalho.

\footnotetext{
${ }^{1}$ Considerando $q^{-1} y(t)=y(t-1)$
}

\section{METOdologia DE PROJeTO DE CONTROLADOR DIGITAL.}

A segunda parte da estratégia de controle adaptativo auto-ajustável indireto proposto neste trabalho é a síntese do sinal de controle. Nesta seção serão apresentadas as duas metodologias de projeto de controlador digital utilizadas para realizar essa tarefa: GPC e alocação de pólos.

\subsection{Generalized Predictive Controlller - GPC}

O algoritmo GPC proposto por Clarke em 1987 (Clarke et al., 1987a), em sua versão incremental, tem o objetivo de minimizar o seguinte critério de desempenho:

$$
\begin{gathered}
J_{G P C I}=E\left\{\sum_{k=N_{1}}^{N_{2}}\left[y(t+k)-y_{d}(t+k)\right]^{2}+\right. \\
\left.\sum_{k=1}^{N_{u}} \lambda(t+k-1)[\Delta u(t+k-1)]^{2}\right\}
\end{gathered}
$$

onde: $y_{d}(t+k)$ é a trajetória desejada para o sinal de saída; $N_{1}$, e $N_{2}$ são os horizontes de predição inicial e final, respectivamente; $N_{u}$ é o horizonte de controle; $\Delta u(t)=u(t)-u(t-1)$ (considera-se que $\Delta u(t+k)=$ $\left.0, k=N_{u}, \ldots, N_{2}\right)$; e $\lambda(j)$ é uma constante que pondera a importância da magnitude das futuras ações de controle em relação ao erro da saída $(\lambda(j)=\lambda$, para $j=t, \ldots t+N_{u}-1$ e $\lambda=\infty$, para $\left.j \geq t+N_{u}\right)$. A estratégia de controle definida em (5) é conhecida como controle incremental, haja vista a inerente ação integral de controle proporcionada pelo operador $\Delta . u(t)$ e $y(t)$ são os sinais de entrada e saída do processo, representado pelo modelo CARIMA representado na Eq. (1).

Para resolver o sistema algébrico (5), é necessário calcular um conjunto de predições de futuras saídas $y(t+j)$ para $j=N_{1}, \ldots, N_{2}$, com base na informação conhecida até o instante $t$ e nos valores futuros dos incrementos do sinal de controle, que serão escolhidos de tal forma a minimizar o critério $J_{G P C I}$. Para calcular as predições da saída, é necessário utilizar, de acordo com a teoria de preditores de modelos estocásticos (Aström and Wittenmark, 1998), um conjunto de equações recorrentes do tipo diophantina. Essas predições podem ser separadas em predições de resposta livre, que são independentes das futuras ações de controle, e predições de resposta forçada, dependentes das futuras ações de controle. As predições livres são agrupadas em um vetor $\mathbf{f}$, de dimensão $N_{2}-N_{1}+1$, definido como:

$$
\mathbf{f}=\left[\hat{y}\left(t+N_{1} \mid t\right), \hat{y}\left(t+N_{1}+1 \mid t\right), \ldots, \hat{y}\left(t+N_{2} \mid t\right)\right]^{T}
$$

sendo $\hat{y}(t+k \mid t), k=N_{1}, \ldots, N_{2}$, as predições de resposta livre de $\hat{y}(t+k)$, dado $\{u(s-1), y(s) ; s \leq t\}$ e supondo 
que $\{u(t+k)=0, k \geq 0\}$. Agora define-se o vetor de futuros incrementos de controle, $\widetilde{\mathbf{u}}$, de dimensão $N_{u}$ :

$$
\widetilde{\mathbf{u}}=\left[\Delta u(t), \Delta u(t+1), \ldots, \Delta u\left(t+N_{u}-1\right)\right]^{T}
$$

e o vetor das predições da saída do processo,

$$
\hat{\mathbf{y}}=\left[\hat{y}\left(t+N_{1}\right), \hat{y}(t+2), \ldots, \hat{y}\left(t+N_{2}\right)\right]^{T}
$$

Utilizando esses vetores, a relação entre os incrementos da entrada e as predições da saída do processo pode ser escrita em notação vetorial como:

$$
\hat{\mathbf{y}}=\mathbf{G} \widetilde{\mathbf{u}}+\mathbf{f}
$$

A matriz $\mathbf{G}$, de dimensão $\left(N_{2}-N_{1}+1\right) \times\left(N_{u}\right)$, é composta pelos parâmetros da resposta ao degrau do modelo (1) considerando o ruído corruptor igual a zero,

$$
\mathbf{G}=\left[\begin{array}{cccc}
g_{N_{1}-1} & 0 & \cdots & 0 \\
g_{N_{1}} & g_{N_{1}-1} & \cdots & 0 \\
\vdots & \vdots & \ddots & \vdots \\
g_{N_{u}-1} & g_{N_{u}-2} & \cdots & g_{N_{1}-1} \\
\vdots & \vdots & & \vdots \\
g_{N_{2}-1} & g_{N_{2}-2} & \cdots & g_{N_{2}-N_{u}}
\end{array}\right]
$$

As predições da resposta livre do modelo, $\hat{y}(t+i \mid t)$, podem ser calculadas iterativamente para todo $i$ a partir da equação (1) considerando $e(t+i)$ e os futuros sinais de controle iguais a zero. $\mathrm{O}$ critério quadrático (5) pode ser reescrito em notação matricial da seguinte forma (Zachariah, 1994), (da Costa Junior, 1999):

$$
J_{G P C I}=(\hat{\mathbf{y}}-\mathbf{r})^{T}(\hat{\mathbf{y}}-\mathbf{r})+\lambda \widetilde{\mathbf{u}}^{T} \widetilde{\mathbf{u}}
$$

onde $\mathbf{r}$ é um vetor de dimensão $N_{2}-N_{1}+1$ contendo os valores da seqüência de futuros sinais de referência:

$$
\mathbf{r}=\left[y_{d}\left(t+N_{1}\right), y_{d}\left(t+N_{1}+1\right), \cdots, y_{d}\left(t+N_{2}\right)\right]^{T}
$$

A solução de (5) é o vetor de incrementos de controle, $\widetilde{\mathbf{u}}$, dado por (Zachariah, 1994):

$$
\widetilde{\mathbf{u}}=\left(\mathbf{G}^{T} \mathbf{G}+\lambda \mathbf{I}\right)^{-1} \mathbf{G}^{T}(\mathbf{r}-\mathbf{f})
$$

$\mathrm{O}$ vetor $\widetilde{\mathbf{u}}$ representa um sinal de tempo discreto formado por uma sequência de sugestões de ações de controle no instante atual e em instantes futuros. Somente o primeiro elemento dessa seqüência de controle, $\Delta u(t)$, precisa ser calculado. A lei de controle a ser executada em cada intervalo de amostragem torna-se a seguinte:

$$
u(t)=u(t-1)+\mathbf{g}^{T}(\mathbf{r}-\mathbf{f})
$$

onde $\mathbf{g}$ é um vetor formado pela primeira linha da matriz $\left(\mathbf{G}^{T} \mathbf{G}+\lambda \mathbf{I}\right)^{-1} \mathbf{G}^{T}$.

\subsubsection{Parâmetros de projeto do controldor GPC}

O critério da Eq. (5) depende da escolha de apenas quatro parâmetros de projeto principais: $N_{1}, N_{2}, N_{u}$ e $\lambda$. Se o atraso de transporte do processo $d$ é conhecido, esse é o valor escolhido para o horizonte inicial de predição da saída $N_{1}$, já que, teoricamente, seria inútil penalizar as saídas que não são afetadas pela ação de controle no instante $t$ (Barreiros et al., 1998). Na prática, o algoritmo funcionará se o atraso real do sistema estiver incluído no intervalo entre $N_{1}$ e $N_{2}$. Usualmente, para o caso em que o atraso é desconhecido, $N_{1}$ é escolhido como a menor estimativa do mesmo. O horizonte de predição $N_{2}$ deve exceder a ordem do polinômio $B$ do modelo do processo. Um valor maior de $N_{2}$, aproximadamente igual ao número de intervalos de amostragens contidos no tempo de subida do processo, é desejado (Clarke et al., 1987). Por outro lado, a penalidade por tal escolha é o aumento considerável da complexidade numérica da solução do problema. O horizonte de controle $N_{u}$ limita o intervalo de tempo em que o sinal de comando tem um valor finito e evita que ele atinja um valor ilimitado. O trabalho original sobre GPC (Clarke et al., 1987), sugere a escolha $N_{u}=1$ para processos simples (aqueles estáveis em malha aberta de fase mínima ou não) e o aumento de $N_{u}$ conforme a complexidade do processo aumente. O trabalho também propõem a escolha de $N_{u}$ igual ao número de pólos mal amortecidos do processo. A escolha de valores pequenos para esse parâmetro é vantajosa sob o ponto de vista computacional pois a dimensão da matriz a ser invertida na Eq. (13) é proporcional a $N_{u}$. A escolha $\lambda=0$ é sugerida originalmente em (Clarke et al. 1987). Sob o ponto de vista numérico, no entanto, a escolha de um valor de pequena magnitude para $\lambda$ é aconselhável.

Os coeficientes do polinômio $C\left(q^{-1}\right)$, quando não são estimados, podem ser escolhidos como parâmetros de projeto do controlador. Em relação ao processo de estimação, esse polinômio pode funcionar como um filtro passa-baixa que reduz o efeito de dinâmicas de alta freqüência sobre o estimador, aumentando sua robustez. Em relação ao controlador, o polinômio $C\left(q^{-1}\right)$ é utilizado como um polinômio observador capaz de melhora a capacidade de rejeição a ruídos do sistema em malha fechada, sem alterações significativas sobre a característica de regulação do controlador. Na prática um polinômio de primeira ordem do tipo $C\left(q^{-1}\right)=1-c_{1} q^{-1}$, é normalmente escolhido. $\mathrm{O}$ valor de $c_{1}$ deve ser compatível com a faixa de freqüências a serem observadas no experimento. Por exemplo, $c_{1}=0,8$ permite que o filtro com função de transferência igual a $1 / C$ tenha uma freqüência de corte de $1,8 H z$, que é apropriada para a observação de alguns tipos de fenômenos eletro- 
mecânicos em sistemas de potência, como por exemplo, modos locais de oscilação que geralmente estão situados na faixa de 0,7 a $2,0 \mathrm{~Hz}$.

\subsection{Técnica de Alocação de Pólos}

Neste trabalho essa metodologia de projeto será utilizada considerando que o processo a ser controlado é representado por um modelo ARX (Auto Regressivo com Entradas Exógenas), que pode ser obtido a partir da equação (1) fazendo $C\left(q^{-1}\right)=\Delta=1$. A seguinte estrutura de canônica de controle RST (Landau, 1993) será utilizada:

$$
R\left(q^{-1}\right) u(t)=T\left(q^{-1}\right) r(t)-S\left(q^{-1}\right) y(t)
$$

onde $R\left(q^{-1}\right), S\left(q^{-1}\right)$ e $T\left(q^{-1}\right)$ são polinômios de ordem apropriadas e $r(t)$ é a trajetória desejada para o sinal de saída $y(t)$. Esse modelo de controlador é muito amplo e aplicável a uma grande variedade de problemas como regulação, rastreamento e rejeição de distúrbios (Aström and Wittenmark, 1998) (Landau, 1993). Neste trabalho, somente o problema da regulação será considerado e, dessa forma, faz-se $T\left(q^{-1}\right)=S\left(q^{-1}\right)$. A técnica de alocação de pólos consiste em encontrar os polinômios $R\left(q^{-1}\right)$ e $S\left(q^{-1}\right)$ que resolvem o seguinte sistema de equações lineares, que representa a dinâmica de malha fechada do processo a ser controlado:

$$
A\left(q^{-1}\right) R\left(q^{-1}\right)+q^{-d} B\left(q^{-1}\right) S\left(q^{-1}\right)=P\left(q^{-1}\right)
$$

Para o caso particular com $d=1$, esse sistema pode ser representado em pela seguinte equação matricial:

$$
\mathbf{M x}=\mathbf{L}
$$

onde:

$$
\begin{aligned}
& \mathbf{M}=\left[\begin{array}{cccccccc}
1 & 0 & \cdots & 0 & b_{0} & 0 & \cdots & 0 \\
a_{1} & 1 & \ddots & \vdots & b_{1} & b_{0} & \cdots & \vdots \\
a_{2} & a_{1} & \cdots & 0 & \vdots & b_{1} & \cdots & \vdots \\
\vdots & \vdots & \cdots & 1 & b_{n_{b}} & \vdots & \cdots & 0 \\
a_{n_{a}} & \vdots & \cdots & a_{1} & 0 & b_{n_{b}} & \cdots & b_{0} \\
0 & a_{n_{a}} & \cdots & \vdots & \vdots & 0 & \cdots & b_{1} \\
\vdots & \vdots & \cdots & \vdots & \vdots & \vdots & \cdots & \vdots \\
0 & 0 & \cdots & a_{n_{a}} & 0 & 0 & \cdots & b_{n_{b}}
\end{array}\right] \\
& \mathbf{x}=\left[\begin{array}{llllllll}
r_{1} & r_{2} & \cdots & r_{n_{r}} & s_{0} & s_{1} & \cdots & s_{n_{s}}
\end{array}\right]^{T} \\
& \mathbf{L}=\left[\left(p_{1}-a_{1}\right) \cdots\left(p_{n_{a}}-a_{n_{a}}\right) \cdots p_{n_{p}} 0 \cdots 0\right]^{T}
\end{aligned}
$$

sendo $p_{1}, p_{2}, \ldots, p_{n_{p}}$ os coeficientes do polinômio que determinam o comportamento desejado em malha fechada.
Os detalhes da escolha desses coeficientes serão apresentados na seção 5.3.1; os termos $s_{i}$ e $r_{j}$, com $i \in\left[1, n_{s}\right]$ e $j \in\left[0, n_{r}\right]$ são os coeficientes dos polinômios $S$ e $R$ da lei de controle (15), que possuem a seguinte estrutura:

$$
\begin{gathered}
S\left(q^{-1}\right)=s_{0}+s_{1} q^{-1}+\cdots+s_{n_{s}} q^{-n_{s}} \\
R\left(q^{-1}\right)=1+r_{1} q^{-1}+r_{2} q^{-2}+\cdots+r_{n_{r}} q^{-n_{r}}
\end{gathered}
$$

\section{RESULTADOS EXPERIMENTAIS}

\subsection{Descrição do Sistema de Geração de Energia de Escala Reduzida - SGER}

O SGER é formado por um gerador síncrono de 10KVA, $220 \mathrm{~V}, 60 \mathrm{hz}, 1200 \mathrm{rpm}$ com pólos salientes acoplada a um motor CC de acionamento de $9 \mathrm{~kW}$. Um volante de aço é utilizado no acoplamento para aumentar a massa inercial do conjunto para simular um sistema de potência de grande porte. Na figura 1, é apresentada a fotografia do sistema. Toda a instrumentação de acionamento, automação e controle está instalada em um armário metálico padrão industrial que se comunica com o grupo motor-gerador e com restante do laboratório por meio das instalações elétricas instaladas em calhas metálicas.

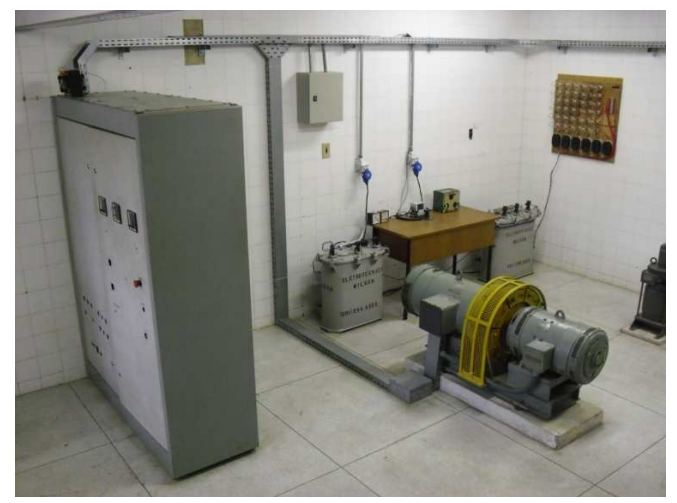

Figura 1: Fotografia do Grupo Gerador e da cadeia de medição e controle.

Na Figura 2 é apresentado o diagrama de blocos do SGER. O sistema de excitação do gerador síncrono é formado por uma ponte retificadora tiristorizada que controla o nível de tensão aplicado ao campo do gerador. A variável $E_{f d}(k)$ representa esse sinal. O RAT utiliza um esquema de controle adaptativo que será detalhado na seção 5.3. O controle de velocidade do motor CC é realizado através do controle do nível de tensão aplicado na armadura do mesmo. O sinal $E_{a}(k)$ representa essa variável. O RV é um controlador com parâmetros fixos, cujo projeto, baseado no método de alocação de polos, foi descrito em (Moutinho, da Costa Jr. and Barra, 2006). 


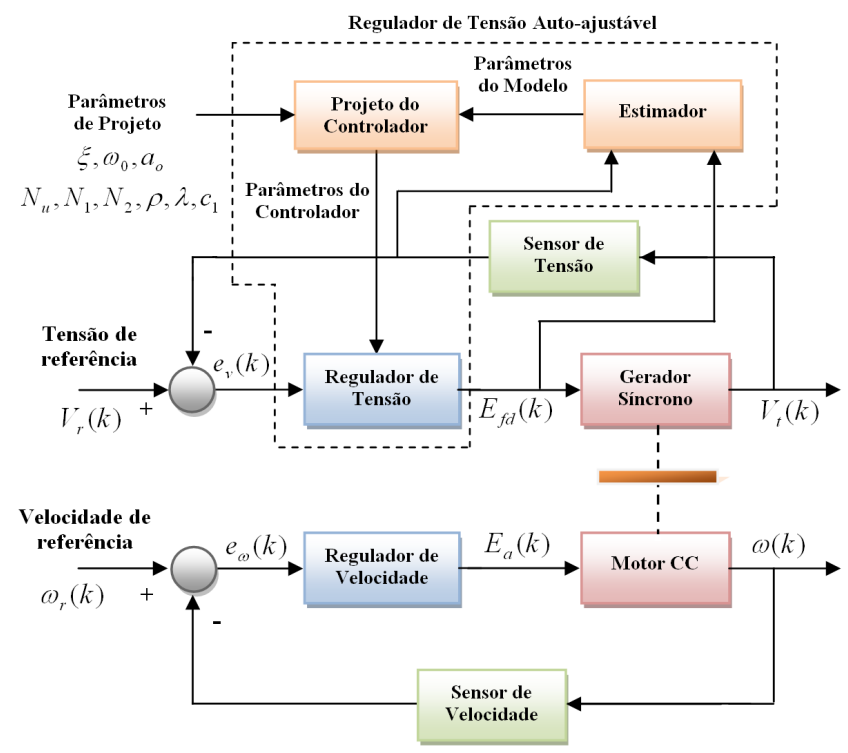

Figura 2: Diagrama de blocos do SGER.

\subsection{Algoritmos adaptativo \\ de Controle Não-}

Para efeito de comparação, todos os testes realizados para medir o desempenho dos controladores adaptativos também serão aplicados à três estratégias de controle não-adaptativo de tensão: controle PID convencional, controle PID Fuzzy e controle por alocação de pólos. Os projetos dos três controladores digitais que utilizam essas estratégias daqui por diante serão identificados, respectivamente, pelas siglas CPID (Controlador PID), CPIDF (Controlador PID Fuzzy) e CDI (Controlador Digital), foram desenvolvidos também pelos autores deste trabalho e são descritos detalhadamente em (Moutinho, da Costa Jr. and Barra, 2006) e (Moutinho, da Costa Jr., Barra and Lima, 2006). No Anexo B são apresentadas as estruturas desses controladores.

\subsection{Algoritmos de Controle Auto-ajustável}

Os dois controladores auto-ajustáveis de tensão propostos neste trabalho serão referenciados da seguinte forma: o controlador auto-ajustável que utiliza o método de alocação de pólos para a síntese do controlador digital de tensão será referenciado como Controlador Adaptativo por Alocação de Pólos (CADAP). O controlador auto-ajustável que utiliza o método de Controle Preditivo Generalizado Incremental (GPCI) para sintetizar o controlador digital de tensão será referenciado como Controlador Adaptativo GPCI (CADGPCI).
A etapa de identificação utilizada nas duas estratégias de controle é realizada pelo ERMQ, descrito na seção 3. Os coeficientes do seguinte modelo ARX de ordem 2 devem ser identificados em tempo real:

$$
\frac{V_{t}(k)}{E_{f d}(k)}=\frac{q^{-1}\left(b_{0}+b_{1} q^{-1}\right)}{1+a_{1} q^{-1}+a_{2} q^{-2}}
$$

sendo $V_{t}(k)$ o sinal de tensão terminal e $E_{f d}(k)$ o sinal de controle da tensão de campo do gerador. Os testes de resposta em freqüência realizados no sistema em estudo demonstraram que uma taxa de amostragem de $20 \mathrm{~ms}$ para a malha de tensão é um valor apropriado.

\subsubsection{Algoritmo CADAP}

O CADAP utiliza esse modelo para resolver o sistema algébrico (17) e formular, a cada intervalo de amostragem, a seguinte lei de controle:

$$
\frac{E_{f d}(k)}{e_{v}(k)}=\frac{s_{0}+s_{1} q^{-1}+s_{2} q^{-2}}{1+r_{1} q^{-1}+r_{2} q^{-2}}
$$

onde $e_{v}(k)$ é o erro discreto entre a tensão terminal, $V_{t}(k)$, e a referência de tensão, $V_{r}(k)$. Para resolver o sistema (17) é necessário escolher o polinômio de malha fechada $P\left(q^{-1}\right)$. Esse polinômio tem a seguinte estrutura:

$$
P\left(q^{-1}\right)=P_{d}\left(q^{-1}\right) A_{o}\left(q^{-1}\right)
$$

onde:

$$
\begin{gathered}
P_{d}\left(q^{-1}\right)=1+p_{d 1} q^{-1}+p_{d 2} q^{-2} \\
p_{d 1}=-2 e^{-\xi \omega_{0} T_{s}} \cos \left(\omega_{0} T_{s} \sqrt{1-\xi^{2}}\right) \\
p_{d 2}=e^{-\xi \omega_{0} T_{s}} \\
A_{o}\left(q^{-1}\right)=\left(1-a_{o} q^{-1}\right)^{2}
\end{gathered}
$$

O polinômio $P\left(q^{-1}\right)$ é, portanto, especificado pelos seguintes parâmetros: $\xi, \omega_{0}$, e $a_{o}$. Esses são os parâmetros de projeto do CADAP. A escolha desses parâmetros foi realizada com base em observações experimentais do comportamento em malha fechada durante testes de resposta ao degrau. Nesses testes, foi observado que, em relação ao parâmetro $\omega_{0}$, quanto maior é o seu valor, maior é o esforço de controle, menor é o tempo de subida e maior é o sobre-sinal da resposta ao degrau do sistema. Em relação ao parâmetro $\xi$, foi observado que, quanto maior é o seu valor, menor é o sobre-sinal. Em relação ao parâmetro $a_{o}$, os testes indicaram que quanto maior é o seu valor, menor é o esforço de controle, maior é o valor do tempo de subida e menor é o valor do sobresinal. Um compromisso de projeto foi, empiricamente, estabelecido para escolher pólos rápidos o suficiente para alcançar a velocidade de resposta desejada sem prejudicar o amortecimento transitório e sem saturar os atuadores do sistema. 


\subsubsection{Algoritmo CADGPCI}

O CADGPCI utiliza os polinômios $A\left(q^{-1}\right)$ e $B\left(q^{-1}\right)$ do modelo Eq. (23), estimados pelo ERMQ, para formular os coeficientes $g_{i}, i=N_{1}-1, N_{1}, \cdots, N_{2}-1$ da resposta ao degrau do seguinte modelo CARIMA:

$$
A\left(q^{-1}\right) \Delta V_{t}(k)=q^{-d} B\left(q^{-1}\right) \Delta E_{f d}(k)+C\left(q^{-1}\right) e(k)
$$

Os coeficientes $g_{i}$ são utilizados para formular a matriz (10), utilizada para resolver o sistema linear (13). Neste trabalho o polinômio $C\left(q^{-1}\right)$ será interpretado como um observador polinomial de coeficientes fixos com a seguinte estrutura $C\left(q^{-1}\right)=1+c_{1} q^{-1}$. Os valores das futuras referências $y_{d}(i), i=k+N_{1}, k+N_{1}+1, \cdots, k+N_{2}$, componentes do vetor (12), são calculados no instante $k$ iterativamente a partir do seguinte sistema de primeira ordem:

$$
y_{d}(k)=(1-\rho) e_{v}(k)+\rho y_{d}(k-1)
$$

onde $\rho$ é uma constante que determina a velocidade com que a saída deve atingir seu valor final. Os parâmetros de projeto necessários para implementar o CADGPCI são: $N_{u}, N_{1}, N_{2}, \rho, \lambda$ e $c_{1}$. A especificação dos valores desses parâmetros de projeto será abordada mais adiante.

\subsection{Testes de Avaliação do Desempenho Dinâmico do RAT Preditivo.}

Os controladores propostos foram implementados em um microcomputador PC AMD K6 II 400Mhz. Uma placa $\mathrm{AD} / \mathrm{DA}$ foi instalada no computador para medir as variáveis tensão terminal e velocidade e enviar os sinais de saída dos controladores digitais das duas malhas de controle para o sistema de energia. No sensor de tensão, a tensão trifásica do estator do gerador síncrono é reduzida através de transdutores de efeito Hall, retificada e filtrada para formar um sinal CC proporcional a tensão terminal. O sensor de velocidade é formado por um tacômetro acoplado ao eixo do gerador que fornece um sinal com freqüência proporcional a sua velocidade de rotação. As rotinas utilizadas nos programas que implementam os controladores foram escritas em linguagem de programação $\mathrm{C}$.

Os testes realizados para avaliar o desempenho dinâmico dos controladores foram os seguintes:

1. variação do tipo degrau na malha de tensão;

2. rastreamento de sinal de referência da malha de tensão:
Todos os testes foram realizados com o sistema de geração operando isolado de rede de energia e inicialmente com tensão e velocidade nominais $(220 \mathrm{~V}$ e $20 \mathrm{~Hz}$, respectivamente), fator de potência unitário e nível de carregamento variável.

\subsubsection{Resultados do Teste 1}

Este teste tem a duração total de $35 \mathrm{~s}$ sendo dividido em 2 partes. Os primeiros 30s são utilizados para estimar os parâmetros do modelo Equ (23), inicializados todos iguais a 0,0001 . Nesse período um sinal do tipo Seqüência Binária Pseudo Aleatória (SBPA) com $100 \mathrm{mV}$ de amplitude, 8 bits e tempo de bit de $70 \mathrm{~ms}$ é superposto ao sinal de controle para acelerar o processo de identificação. O ERMQ é inicializado com fator de esquecimento fixo em 0,99 e matriz de covariância $10^{6} I$, onde $I$ representa uma matriz identidade de ordem apropriada. $\mathrm{O}$ algoritmo de síntese do controlador é inicializado em $t=0 \mathrm{~s}$, simultaneamente com o início do processo de estimação. Durante o transitório inicial um limitador do sinal de controle é utilizado para evitar uma perturbação muito grande no valor da tensão terminal. Nos 5 últimos segundos do teste o estimador é paralisado, o sinal SBPA é interrompido e um degrau é aplicado na referência de tensão.

Na Figura 3 são apresentados os resultados obtidos com o CADAP no teste. Os parâmetros de projeto do controlador foram especificados em $\xi=0,8, \omega_{0}=7 \mathrm{rad} / \mathrm{s}$, e $a_{o}=0$. Durante os primeiros 5 segundos do teste o sinal de controle foi limitado em $\pm 0,5 \mathrm{~V}$. A convergência dos coeficientes do polinômio $S$ do controlador ocorre após uma fase transitória observada nos primeiros segundos do teste. A limitação do sinal de controle atenuou as variações desse transitório permitindo a rápida convergência dos parâmetros do controlador.

Na Figura 4 são apresentados os resultados obtidos com o CADGPCI durante o teste. Os parâmetros de projeto do controlador foram especificados em $N_{1}=1, N_{2}=8$, $N_{u}=2, \lambda=0,3, \rho=0,1$ e $\left.c_{1}=0\right)$ Como o modelo matemático da Eq. (23) utilizado para representar a malha de tensão é do tipo ARX, os coeficientes do polinômio $C\left(q^{-} 1\right)$ não precisam ser estimados. Nesse trabalho não foi realizado um estudo sobre os efeitos que o polinômio $\mathrm{C}$ exerce sobre a capacidade de rejeição de distúrbios do CADGPCI no teste 1. Por isso foi escolhido $c 1=0$. Verifica-se pela figura a rápida convergência dos parâmetros do modelo devido à utilização do sinal SBPA na primeira parte do teste. Na Tabela 1, são resumidos os valores dos índices de desempenho obtidos no teste. Os dois controladores adaptativos conseguiram responder adequadamente ao teste exibindo baixo sobre-sinal e 

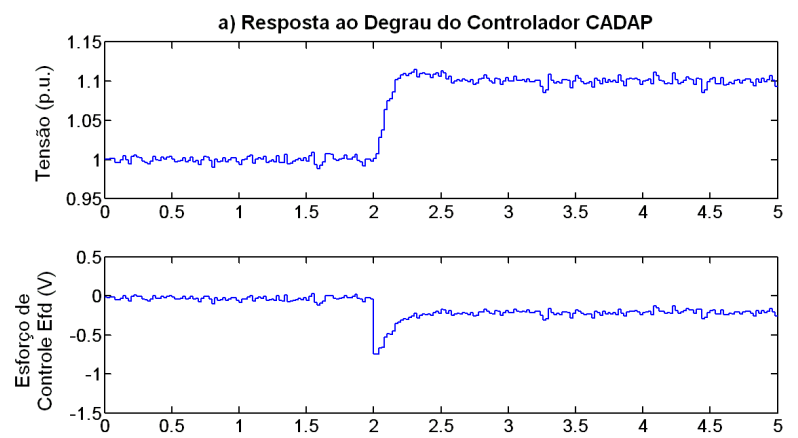

b) Parâmetros do Controlador S(q-1)/R(q-1)
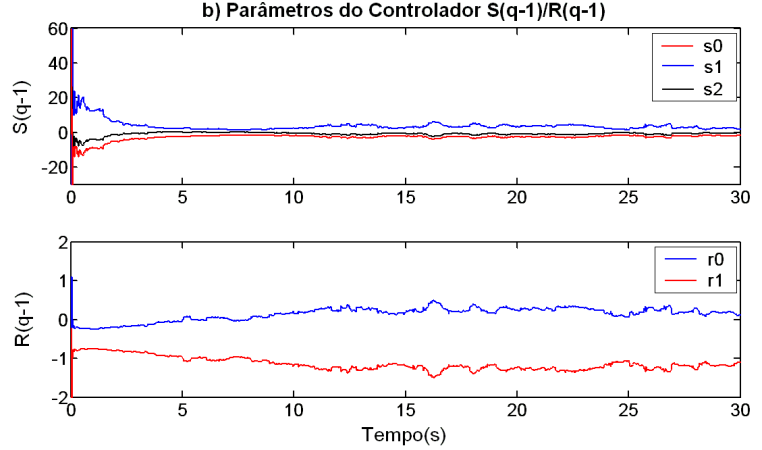

Figura 3: Resultados obtidos com o CADAP no teste 1. Degrau de 0,1p.u. de amplitude e sistema com carga de 1200W.
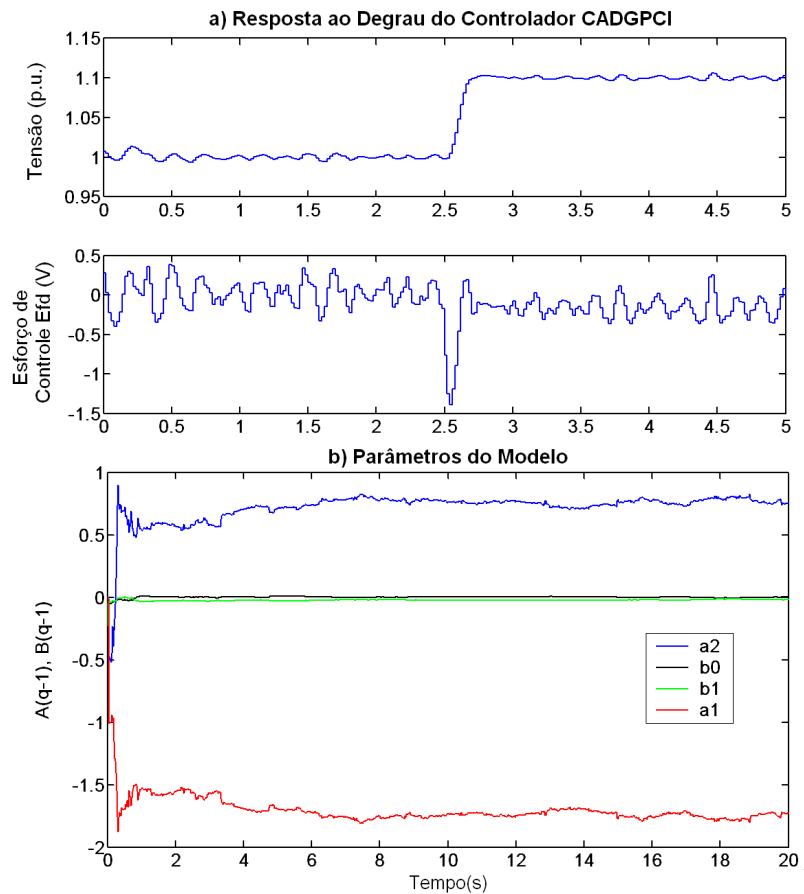

Figura 4: Resultados obtidos com o controlador CADGPCI no teste 1. Degrau de 0,1p.u. de amplitude carregamento de $1200 \mathrm{~W}$

boa velocidade de resposta. Na Tabela 2 , são resumidos
Tabela 1: Desempenho dos Controladores Adaptativos no Teste 1 ( $t_{s}$ - tempo de estabilização; $t_{r}$ - tempo de subida; A - Amplitude; C - Carga; SS - Sobre-sinal).

\begin{tabular}{c|c|c|c|c}
\hline \hline $\mathrm{A}(\%)$ & $\mathrm{C}(\mathrm{kw})$ & $t_{s}(s)$ & $t_{r}(s)$ & $\mathrm{SS}(\%)$ \\
\hline \hline \multicolumn{5}{c}{ CADAP } \\
\hline \hline 5 & 0 & 0,36 & 1,69 & 16 \\
\hline 5 & 1,2 & 0,42 & 0,78 & 20 \\
\hline 5 & 2,4 & 0,4 & 0,75 & 12,5 \\
\hline 10 & 0 & 0,38 & 1,95 & 13 \\
\hline 10 & 1,2 & 0,42 & 1,04 & 14,7 \\
\hline 10 & 2,4 & 0,44 & 1,08 & 13,75 \\
\hline \hline \multicolumn{5}{c}{ CADGPCI } \\
\hline \hline 5 & 0 & 0,1 & 0,1 & 0 \\
\hline 5 & 1,2 & 0,28 & 0,1 & 10 \\
\hline 5 & 2,4 & 0,1 & 0,1 & 0 \\
\hline 10 & 0 & 0,1 & 0,1 & 0 \\
\hline 10 & 1,2 & 0,1 & 0,1 & 0 \\
\hline 10 & 2,4 & 0,08 & 0,08 & 0 \\
\hline \hline
\end{tabular}

os valores dos índices de desempenho obtidos no teste com os controladores não-adaptativos.

Os resultados médios obtidos no teste 1 com o CADAP e o CADGPCI são, na maioria dos casos, superiores aos resultados obtidos com os controladores digitais nãoadaptativos CDI, CPID e CPIDF, mencionados na Seção 5.2. O CADAP exibe menores tempos de subida e estabilização com apenas um pequeno aumento não significativo no sobre-sinal em relação aos controladores não-adaptativos. Já o CADGPCI foi muito superior ao CADAP e aos controladores não-adaptativos em todos os índices de desempenho analisados e especialmente em relação ao sobre-sinal.

No teste 1 os dois esquemas de controle adaptativo se comportam como controladores a parâmetros fixos no momento da aplicação da perturbação em degrau. Isso acontece porque o esquema de identificação recursiva do CADP e do CAGPCI foi desabilitado nos últimos $5 \mathrm{~s}$ do teste. Os primeiros 30s do teste são utilizados para sintonizar os parâmetros dos controladores adaptativos. Uma vantagem da utilização de um esquema adaptativo nesse contexto é que a sintonia da malha de controle pode ser realizada de forma automática, injetando-se na malha principal uma perturbação SBPA de pequena magnitude. Em ambientes industriais, se o número de parâmetros de sintonia é elevado esse tipo de procedimento pode levar a uma redução do tempo de ajustes, 
Tabela 2: Desempenho dos Controladores não-adaptativos no Teste 1.

\begin{tabular}{c|c|c|c|c}
\hline \hline $\mathrm{A}(\%)$ & $\mathrm{C}(\mathrm{kw})$ & $t_{s}(s)$ & $t_{r}(s)$ & $\mathrm{SS}(\%)$ \\
\hline \hline \multicolumn{5}{c}{ CDI } \\
\hline \hline 5 & 0 & 0,38 & 0,13 & 15 \\
\hline 5 & 1,2 & 0,46 & 0,15 & 16 \\
\hline 5 & 2,4 & 0,46 & 0,1 & 13,75 \\
\hline 10 & 0 & 0,5 & 0,14 & 11,87 \\
\hline 10 & 1,2 & 0,6 & 0,15 & 15 \\
\hline 10 & 2,4 & 0,56 & 0,14 & 11,25 \\
\hline \hline \multicolumn{5}{c}{ CPIDI } \\
\hline \hline 5 & 0 & 0,36 & 0,12 & 13 \\
\hline 5 & 1,2 & 0,45 & 0,12 & 14 \\
\hline 5 & 2,4 & 0,5 & 0,12 & 11,5 \\
\hline 10 & 0 & 0,44 & 0,12 & 8,6 \\
\hline 10 & 1,2 & 0,44 & 0,12 & 8,1 \\
\hline 10 & 2,4 & 0,42 & 0,1 & 10 \\
\hline \hline \multicolumn{5}{|c}{ CPIDF } \\
\hline \hline 5 & 0 & 0,41 & 0,11 & 14,25 \\
\hline 5 & 1,2 & 0,43 & 0,1 & 13 \\
\hline 5 & 2,4 & 0,51 & 0,14 & 19 \\
\hline 10 & 0 & 0,46 & 0,11 & 12,5 \\
\hline 10 & 1,2 & 0,42 & 0,14 & 10 \\
\hline 10 & 2,4 & 0,53 & 0,15 & 14,3 \\
\hline \hline \multicolumn{5}{|c}{} \\
\hline \hline 5
\end{tabular}

além de facilitar o procedimento de sintonia dos parâmetros no caso de degradação de desempenho comprovadamente atribuída a alterações nas características do processo controlado.

\subsubsection{Resultados do Teste 2}

Este teste tem duração total de $75 \mathrm{~s}$ particionados em 2 etapas. Os primeiros 15 segundos são utilizados para estimar os parâmetros do modelo Equ (23), inicializados todos iguais a 0,0001 . Neste período um sinal SBPA similar ao utilizado no teste 1 é superposto ao sinal de controle. O ERMQ é inicializado com fator de esquecimento fixo em 0,98 e matriz de covariância $10^{5} I$. Nesse teste foi verificada experimentalmente a necessidade de reduzir a importância dos dados antigos sobre a atualização dos parâmetros do modelo e, por isso, o fator de esquecimento utilizado foi de 0,98 . O esquema de limitação de esforço de controle descrito no teste anterior foi utilizado. Na etapa 2 do teste, os últimos $60 \mathrm{~s}$, o estimador continua funcionando mas o sinal SBPA tem sua amplitude reduzida. Uma seqüência de variações do tipo degrau é aplicada à referência do controlador de tensão enquanto cargas são inseridas no sistema. As inclusões são de $1,2 \mathrm{~kW}$ e ocorrem em aproximadamente $t=20 \mathrm{~s}$ e $t=40 \mathrm{~s}$ da segunda etapa do teste. $\mathrm{O}$ objetivo do teste é verificar a capacidade de adaptação e convergência do controlador quando o sistema opera em diferentes condições dinâmicas.

Para mensurar o desempenho do controlador de tensão durante este teste o seguinte índice de desempenho quadrático deve ser minimizado:

$$
J_{v}=\sum_{k=0}^{n}\left[V_{r}(k)-V_{t}(k)\right]^{2}
$$

onde $n$ é o número total de amostras, obtidas com o intervalo de amostragem de $\Delta T_{v}=20 \mathrm{~ms}$ utilizado na malha de tensão. O desempenho do controlador de velocidade também é investigado com o auxílio do seguinte índice de desempenho quadrático:

$$
J_{\omega}=\sum_{k=0}^{n}\left[\omega_{r}(k)-\omega(k)\right]^{2}
$$

onde as amostras são obtidas a cada $\Delta T_{\omega}=130 \mathrm{~ms}$, o intervalo de amostragem do controlador de velocidade; $\omega(k)$ e $\omega_{r}(k)$ são os sinais discretos de velocidade e de referência de velocidade, respectivamente.

Na Figura 5 são apresentados os resultados obtidos com o CADAP no teste. Os parâmetros de projeto do controlador foram especificados em $\xi=0,8, \omega_{0}=7 \mathrm{rad} / \mathrm{s}$ e $a_{o}=0$. O limitador de esforço de controle foi utilizado nos 5 primeiros segundos do teste com limites de $\pm 0,5 \mathrm{~V}$. O ruído observado no sinal de saída é causado pelo sinal SBPA de $40 \mathrm{mV}$ de amplitude utilizado na segunda parte do teste para suprir os requisitos de persistência de excitação do mecanismo de identificação.

Na Figura 6 são apresentados os resultados obtidos com o CADGPCI no teste. Os parâmetros de projeto do controlador foram especificados em $N_{1}=1, N_{2}=8$, $N_{u}=2, \lambda=0,3, \rho=0,1$ e $c_{1}=0$. Observações experimentais revelaram que um sinal SBPA de $25 \mathrm{mV}$ de amplitude é suficiente para a manutenção do processo de identificação do sistema durante a segunda parte do teste. Na Tabela 3, são apresentados os resultados dos controladores adaptativos no teste. Os resultados obtidos com os controladores não-adaptativos no teste 2 são apresentados na Tabela 4.

A análise de desempenho dos controladores no teste 2 deve levar em consideração os seguintes aspectos:

1. A amplitude das perturbações SBPA é diferente nos controladores CADAP e CADGPCI, $40 \mathrm{mV}$ e $25 \mathrm{mV}$, respectivamente. A redução da amplitude da SBPA do CADGPCI possibilitou a diminuição 

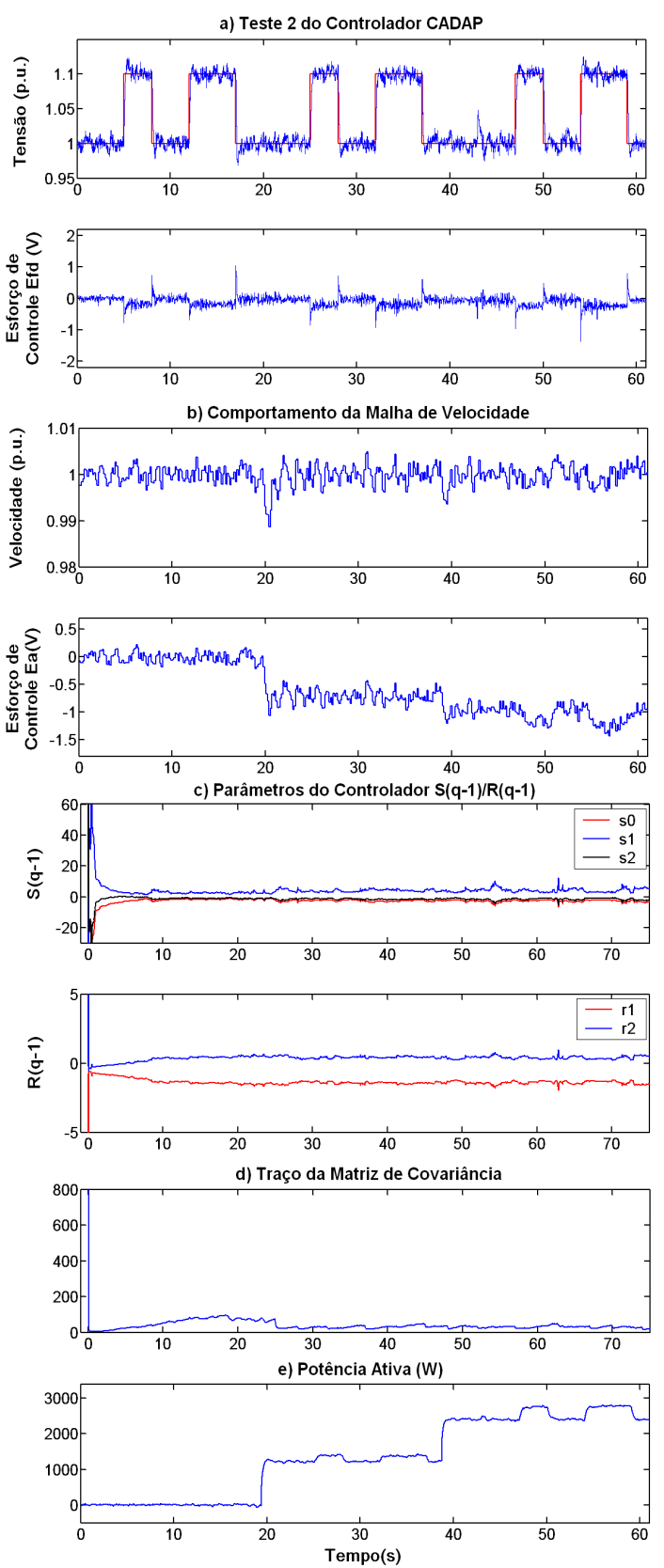

Figura 5: Resultados do CADAP no teste 2.

do erro em regime após a aplicação de cada degrau (comparar com o teste do CADAP onde foi utilizado um SBPA de $40 \mathrm{mV}$ ) e resultou em melhoria no processo de rastreamento contribuindo para reduzir o índice $J_{v}$.

2. O traço da matriz de covariância apresentou maiores magnitudes no CADGPCI. Os valores dos parâmetros identificados para o sistema em malha fechada sofrem variações acentuadas nos instantes em
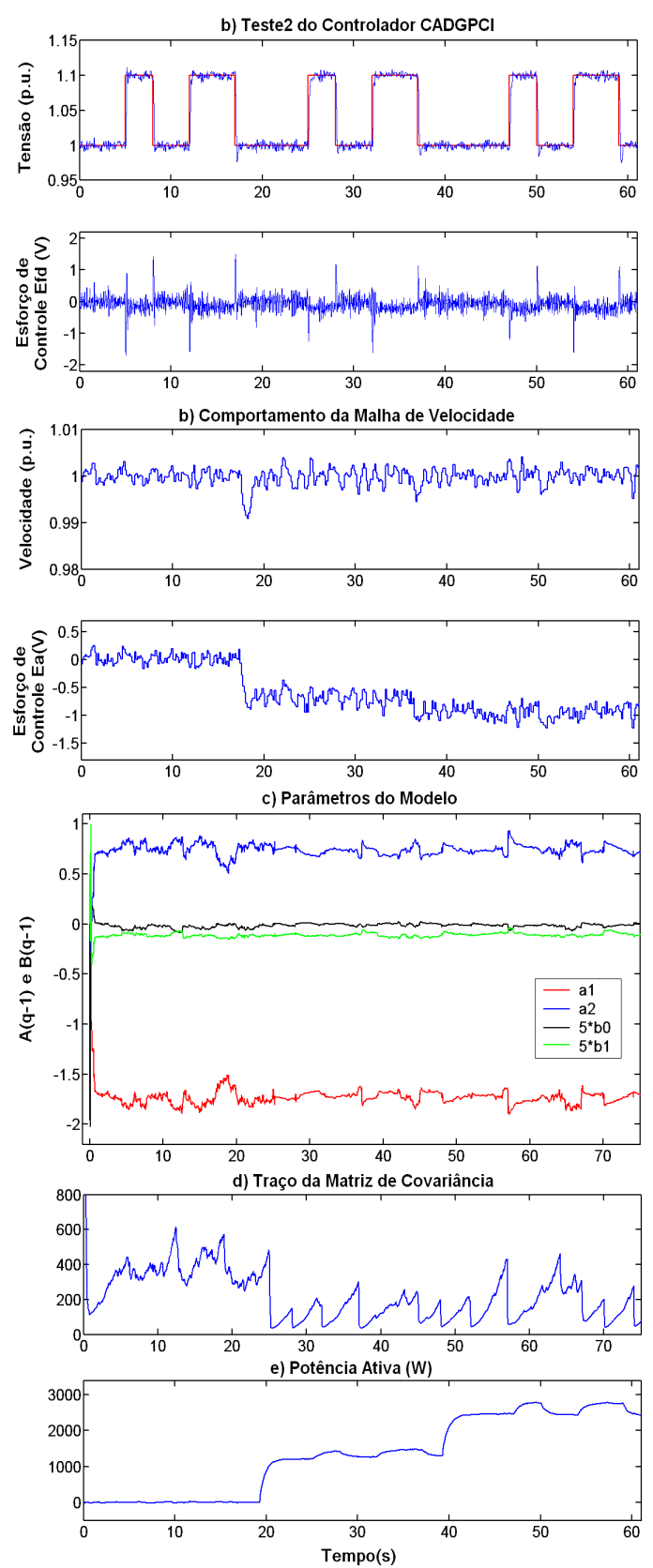

Figura 6: Resultados do CADGPCI no teste 2.

que ocorrem as mudanças na referência de tensão. Nos intervalos em que os valores médios dos parâmetros variam pouco ocorre o aumento do traço da matriz de covariância. Conforme pode ser observado, durante os transitórios do sinal de tensão o valor do traço volta a diminuir. Esse comportamento é atribuído a utilização de uma SBPA de amplitude mais baixa no CADGPCI que limitou a variabilidade da tensão e reduziu a quantidade 
Tabela 3: Desempenho dos Controladores Adaptativos no Teste 2.

\begin{tabular}{c|c|c|c|c}
\hline \hline $\mathrm{A}(\%)$ & $J_{\omega}$ & $J_{v}$ & $\omega_{\max }(p . u)$. & $\omega_{\min }(p . u)$. \\
\hline \hline \multicolumn{5}{c}{ CADGPCI } \\
\hline \hline 5 & 0.19094 & 0.00141 & 1.0043 & 0.9906 \\
\hline 10 & 0.51318 & 0.00184 & 1.0041 & 0.9909 \\
\hline \hline \multicolumn{5}{c}{ CADAP } \\
\hline \hline 5 & 1.42821 & 0.00211 & 1.0056 & 0.9905 \\
\hline 10 & 1.61131 & 0.00235 & 1.0049 & 0.9886 \\
\hline \hline
\end{tabular}

Tabela 4: Desempenho dos Controladores Não-Adaptativos no Teste 2 .

\begin{tabular}{c|c|c|c|c}
\hline \hline $\mathrm{A}(\%)$ & $J_{\omega}$ & $J_{v}$ & $\omega_{\max }(p . u)$. & $\omega_{\min }(p . u)$. \\
\hline \hline \multicolumn{5}{c}{ CDI } \\
\hline \hline 5 & 0,14148 & 0,00854 & 1,0025 & 0,9805 \\
\hline 10 & 0,53048 & 0,00736 & 1,0026 & 0,9811 \\
\hline \hline \multicolumn{5}{c}{ CPID } \\
\hline \hline 5 & 0,09495 & 0,00721 & 1,002 & 0,9812 \\
\hline 10 & 0,33781 & 0,00721 & 1,003 & 0,9817 \\
\hline \hline 5 & 0,11105 & 0,00928 & 1,003 & 0,9781 \\
\hline 10 & 0,37107 & 0,00558 & 1,0031 & 0,9811 \\
\hline \hline
\end{tabular}

de informação dinâmica disponível ao ERMQ resultando em aumento da sensibilidade do estimador. Esse nível de sensibilidade foi considerado adequado para a aplicação analisada neste trabalho já que não resultou em instabilidade numérica em malha fechada e proporcionou uma identificação recursiva coerente com as observações realizadas de forma não-recursiva.

3. O traço da matriz de covariância do CADAP permanece aproximadamente constante na maior parte da segunda etapa do teste, indicando a disponibilidade de informação dinâmica suficiente para a manutenção do modelo do processo. Nota-se também que, para o traço da matriz de covariância quando se utiliza o CADAP, observam-se menores magnitudes e a variabilidade dos parâmetros do controlador é baixa.

4. Os controladores CDI, CPID e CPIDF não utilizam perturbações SBPA em suas malhas isso reduz a variabilidade do sinal de saída e contribui para reduzir o índice de desempenho $J_{v}$, o que é desejável;
Considerando esses aspectos, segue a análise dos resultados do teste 2 :

1. O desempenho do CADGPCI foi superior ao do CADAP em termos de minimização dos critérios quadráticos $J_{v}$ e $J_{\omega}$. A utilização de uma SBPA com amplitude reduzida no CADGPCI resultou em aumento na variação dos parâmetros do modelo e redução da variabilidade do sinal de saída. A utilização de um modelo de previsão que descreve o processo em um horizonte de tempo móvel possibilita que o CADGPCI tolere variações nos parâmetros do modelo sem degradar consideravelmente o desempenho em malha fechada. Essa é uma característica comum aos controladores MPC.

2. O CADGPCI teve desempenho superior a todos os controladores digitais não adaptativos em termos de minimização do critério quadrático $J_{v}$.

3. A análise da tabela 4 revela que os controladores CDI, CPID e CPIDF apresentaram desempenho semelhante no teste 2 .

4. A perda de desempenho do CADAP em relação ao CADGPCI quando se considera a minimização do critério quadrático $J_{v}$ é atribuida à perturbação de tensão provocada pela presença de um sinal SBPA com amplitude de $40 \mathrm{mV}$ superposto ao sinal de controle. Verificou-se experimentalmente que não foi possível reduzir a amplitude desse sinal sem prejudicar o processo de identificação recursiva do CADAP.

5. A flutuação inicial observada na convergência dos parâmetros do modelo pouco influenciou no desempenho dos controladores CADAP e CADGPCI.

6. Em relação ao ERMQ utilizado no teste 2, são observadas variações nos parâmetros do modelo e do controlador conforme a condição operacional é alterada. Conforme apresentado na figura 6 , alterações na condição operacional no teste 2 (inclusão de cargas em $t=20$ s e $t=40$ s e mudanças no nível de tensão terminal) resultam em alterações nos parâmetros identificados recursivamente. Essas alterações também são observadas nos parâmetros do controlador conforme apresentado na figura 5, principalmente quando são considerados os parâmetros $s_{0}$, $s_{1}$ e $s_{2}$. Com base nessas observações experimentais, os autores consideram que a utilização de um esquema de identificação recursiva contribui para melhorar o desempenho dos controladores CADAP e CADGPCI já que permite que esses controladores se adaptem melhor às variações paramétricas do sistema controlado. 
7. Durante os testes experimentais, foi verificado que selecionando valores de $\beta$ na faixa de 0,97 a 0,99 o traço da matriz de covariância assumia valores estáveis ao longo dos ensaios. Valores fora dessa faixa resultaram em instabilidade numérica caracterizada pela explosão do traço da matriz de covariância. Neste trabalho o fator de esquecimento foi selecionado de forma empírica. Embora existam técnicas analíticas para a seleção desse parâmetro tal estratégia ficou fora do escopo do presente trabalho.

8. Foi verificado experimentalmente no teste 2 que valores de $c_{1}$ na faixa de 0 a 0,9 não resultaram em ganho significativo de desempenho do controlador. Conforme o valor de $c_{1}$ aumenta dentro dessa faixa, verificou-se uma redução na variabilidade da resposta já que o polinômio $\mathrm{C}$ funciona como um filtro passa-baixas além de aumentar a capacidade de rejeição de distúrbios, o que reduz os efeitos da perturbação SBPA sobre o sinal de saída. A diminuição da quantidade de informação dinâmica disponível ao ERMQ resultou em aumento dos valores do traço da matriz de covariância, prejudicando o processo de estimação dos parâmetros do modelo. Por essa razão foi utilizado um valor de $c_{1}=0$ no teste 2. Está fora do escopo deste trabalho encontrar a especificação ideal do polinômio C de forma a obter um melhor desempenho do CADAGPCI.

\section{CONCLUSÃO}

Os dois controladores adaptativos projetados neste trabalho tiveram seu desempenho avaliado para testes de resposta ao degrau e rastreamento realizados em diferentes condições de operação. Verificou-se, experimentalmente, que os referidos controladores apresentaram bom desempenho em todos os testes realizados apresentando, tempos de resposta aceitáveis e boa robustez. A comparação entre esses resultados e os resultados obtidos com os controladores digitais não-adaptativos projetados em artigos anteriores revelou que os controladores CADAP e CADGPCI propostos neste artigo tiveram desempenho equivalente ou superior ao desempenho de controladores fixos na maioria das situações contempladas. As técnicas de controle digital avançado mostraram-se mais eficientes e apropriadas no trato das peculiares características não-lineares do sistema de potência estudado.

Os resultados promissores deste trabalho motivam os seus autores a investigar com maior profundidade os seguintes aspectos do controlador MPC no SGER: 1) as possíveis melhorias que podem ser obtidas com a utilização de estratégias de controle preditivo multivariável que levem em consideração as interações existentes entre as malhas desse sistema de potência; 2) estudos detalhados sobre a robustez, rejeição de perturbações e/ou atenuação de ruídos do controlador preditivo; Os autores esperam que em uma próxima oportunidade os resultados desses estudos e da análise do desempenho do MPC multivariável sejam reportados.

\section{ANEXO A - ESTRUTURA DOS CONTRO- LADORES NÃO-ADAPTATIVOS DE TEN- SÃO}

O controlador de tensão PID convencional (denominado CPID) possui a seguinte estrutura:

$$
C P I D=\frac{E_{f d}(k)}{e_{v}(k)}=K \frac{\left(q-z_{c 1}\right)\left(q-z_{c 2}\right)}{q(q-1)}
$$

A sintonia desse controlador foi realizada utilizando o método do lugar geométrico das raízes (LGR). O Controlador digital de tensão (denominado CDI) possui a seguinte estrutura:

$$
C D I=\frac{E_{f d}(k)}{e_{v}(k)}=\frac{r_{0}+r_{1} q^{-1}+r_{2} q^{-2}}{\left(1-q^{-1}\right)\left(1+s_{1} q^{-1}\right)}
$$

A sintonia desse controlador foi realizada utilizando o método de alocação de pólos descrito na seção 4.2. Um modelo matemático simplificado da malha de tensão foi utilizado no processo de sintonia dos controladores CDI e CPID. A estrutura do modelo é apresentada na Eq. (23). Os parâmetros desse modelo foram estimados através de ensaios de identificação.

Na Figura 7 é apresentada a estrutura do controlador PID Fuzzy de tensão (denominado CPIDF). A unidade de lógica Fuzzy utiliza fuzzificador do tipo singleton, máquina de inferência máximo-produto e defuzzificador por centro de gravidade. A sintonia do contralador CPIDF consiste em ajustar os ganhos $G_{e}^{v}, G_{u}^{v}, G_{d e}^{v}$ e $G_{i}^{v}$. O ajuste desses parâmetros foi realizado utilizando métodos empíricos e conhecimentos qualiativos do funcionamento do SGER.

\section{REFERÊNCIAS}

Aguirre, L. A. (2004). Introdução à Identificação de Sistemas, $2^{\circ}$ edn, UFMG.

Aström, K. J. and Wittenmark, B. (1995). Adaptive Control, $2^{\circ}$ edn, Addison Wesley.

Aström, K. J. and Wittenmark, B. (1998). Computer Controlled Systems, $3^{\circ}$ edn, Prentice-Hall. 


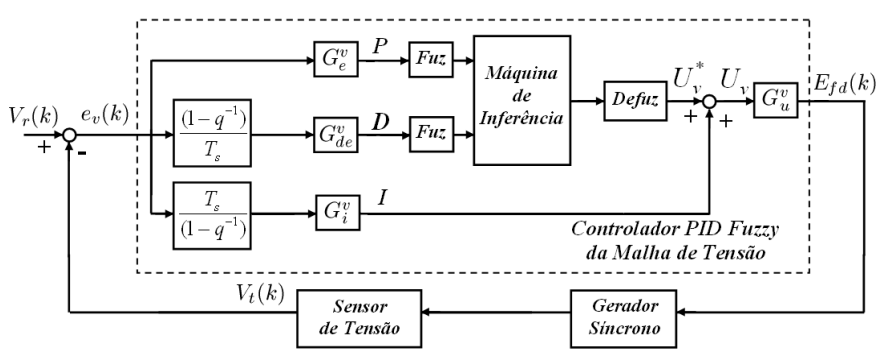

Figura 7: Controlador PID Fuzzy de Tensão.

Barra, W., Barreiros, J. A. L., da Costa Jr., C. T. and Ferreira, A. M. D. (2005). Controle fuzzy aplicado à melhoria da estabilidade dinâmica em sistemas elétricos de potência, Controle $\mathscr{E}$ Automação, Revista da Sociedade Brasileira de Automatica 16(2): 173186.

Bravo, C. O. A. and Normey-Rico, J. E. (2009). Controle de plantas não lineares utilizando controle preditivo linear baseado em modelos locais, Sba Controle $\&$ Automação 20(4): 465-481.

Camacho, E. F. and Bordons, C. (2004). Model Predictive Control, Springer-Verlag.

Clarke, D. W., Mohtadi, C. and Tuffs, P. S. (1987a). Generalized predictive control-part i: The basic algorithm, Automatica 23(2): 137-149.

Clarke, D. W., Mohtadi, C. and Tuffs, P. S. (1987b). Generalized predictive control-part ii: extensions and interpretations, Automatica 23(2): 149-160.

Clarke, D. and Zhang, L. (1987). Long-range predictive control using weighting-sequence models, Control Theory and Applications, IEE Proceedings D 134(3): 187-195.

da Costa Junior, C. T. (1999). Méthodes de commande adaptative par supervision pour la régulation d'un générateur entraîné par turbine hydraulique.

Fontes, C. H. and Mendes, M. J. (2009). Nonlinear predictive control of an industrial slurry reactor, $S b a$ Controle \& Automação 19(4): 417-430.

Landau, I. D. (1993). Identification et commande de Systemes, deuxième edn, Hermes.

Larsen, E. V. and Swann., D. A. (1981). Applying power systems stabilizers (parts 1, 2, 3), IEEE Trans. Power Appar. Syst PAS-100(6): 3017-3024, 30253033, 3034-3046.
Moutinho, M. N., da Costa Jr., C. T. and Barra, W. (2006). Experimental results in identification and integrated digital control in a synchronous machine, XVI Automatic Brazilian Congress (CBA), Salvador, Brazil, .

Moutinho, M. N., da Costa Jr., C. T., Barra, W. and Lima, M. N. d. (2006). Resultados experimentais em controle em tempo real pid fuzzy integrado de uma máquina síncrona, XVI Congresso Brasileiro de Automática (CBA), Salvador, Brasil .

Robak, S. (2009). Robust svc controller design and analysis for uncertain power systems, Control Engineering Practice, Elsevier .

Sauer, P. and Pai, M. (1998). Power system dynamic and stability, Prentice-Hall: Englewood Cliffs.

Zachariah, K. J. (1994). Implementation of Self-Tuning Control for Turbine Generators, Phd thesis, Department of Electrical and Electronic Engineering, University of Newcastle-upon-Type, UK. 